\title{
Aerodynamic Analysis of Simulated Heat Shield Recession for the Orion Command Module
}

\author{
Karen L. Bibb, Stephen J. Alter ${ }^{\dagger}$ \\ NASA Langley Research Center, Hampton, VA 23681 \\ Ryan McDaniel ${ }^{\ddagger}$ \\ NASA Ames Research Center, Moffet Field, CA \\ Corresponding author: karen.l.bibb@nasa.gov \\ phone: +17578648005, fax: +17578648670 \\ Submitted to 46th AIAA Aerosciences Meeting and Exhibit \\ January, 2008/Reno, NV
}

\begin{abstract}
The aerodynamic effects of the recession of the ablative thermal protection system for the Orion Command Module are important for the vehicle guidance. At the present time, the aerodynamic effects are being handled within the Orion Aerodatabase as an additional uncertainty which is not based on strong analysis. This study is an initial attempt to quantify the effects for a particular set of recessed geometry shapes, in order to either reduce or justify the current aerodatabase uncertainties. The aerodynamics for the baseline and recessed geometries were computed at several trajectory points using multiple CFD codes, both viscous and inviscid. The resulting aerodynamics for the baseline and recessed geometries were compared. The forces $\left(C_{L}, C_{D}\right)$ showed negligible differences between baseline and recessed. Generally, moments showed a consistent delta between baseline and recessed, and correlate with the maximum amount of recession of the geometry. $\Delta C_{m}$ increases as Mach number decreases (and the recession is greater). The change in trim angle of attack increases from $\sim 0.5^{\circ}$ at $U_{\infty}=9 \mathrm{~km} / \mathrm{s}$ to $\sim 1.3^{\circ}$ at $M=6$, and is consistent with previous analysis with a lower fidelity engineering tool. The present analysis suggests that while the effect is handled with the current database uncertainty bounds, we cannot use this data to reduce the database uncertainties, and that we will need to revisit the issue when the trajectories to be flown are better defined, and as we move closer to lunar missions and begin considering Mars missions.
\end{abstract}

\section{Abstract Submittal Notes}

This submittal to the 46th AIAA Aerosciences Meeting in January, 2008 represents a body of work recently completed under the Orion / CEV Aerosciences Program. The report for the work is in final edit, but has not been included in this submission as a final determination on ITAR restrictions has not been made.

* Research Engineer, Aerothermodynamics Branch, Senior Member AIAA

${ }^{\dagger}$ Research Engineer, Aerothermodynamics Branch, Member AIAA

¥Research Scientist, Member AIAA

This material is declared a work of the U.S. Government and is not subject to copyright protection in the United States. 


\title{
Aerodynamic Analysis of Simulated Heat Shield Recession for the Orion Command Module
}

\author{
Karen L. Bibb, Stephen J. Alter ${ }^{\dagger}$ \\ NASA Langley Research Center, Hampton, VA 23681 \\ Ryan McDaniel ${ }^{\ddagger}$ \\ NASA Ames Research Center, Moffet Field, CA 94035
}

\begin{abstract}
The aerodynamic effects of the recession of the ablative thermal protection system for the Orion Command Module of the Crew Exploration Vehicle are important for the vehicle guidance. At the present time, the aerodynamic effects of recession being handled within the Orion aerodynamic database indirectly with an additional safety factor placed on the uncertainty bounds. This study is an initial attempt to quantify the effects for a particular set of recessed geometry shapes, in order to provide more rigorous analysis for managing recession effects within the aerodynamic database. The aerodynamic forces and moments for the baseline and recessed geometries were computed at several trajectory points using multiple CFD codes, both viscous and inviscid. The resulting aerodynamics for the baseline and recessed geometries were compared. The forces (lift, drag) show negligible differences between baseline and recessed geometries. Generally, the moments show a difference between baseline and recessed geometries that correlates with the maximum amount of recession of the geometry. The difference between the pitching moments for the baseline and recessed geometries increases as Mach number decreases (and the recession is greater), and reach a value of -0.0026 for the lowest Mach number. The change in trim angle of attack increases from $\sim 0.5^{\circ}$ at $M=28.7$ to $\sim 1.3^{\circ}$ at $M=6$, and is consistent with a previous analysis with a lower fidelity engineering tool. This correlation of the present results with the engineering tool results supports the continued use of the engineering tool for future work. The present analysis suggests there does not need to be an uncertainty due to recession in the Orion aerodynamic database for the force quantities. The magnitude of the change in pitching moment due to recession is large enough to warrant inclusion in the aerodynamic database. An increment in the uncertainty for pitching moment could be calculated from these results and included in the development of the aerodynamic database uncertainty for pitching moment.
\end{abstract}

\section{Nomenclature}

$C_{D} \quad$ Drag coefficient

$C_{L} \quad$ Lift coefficient

$C_{m} \quad$ Pitching moment coefficient

D Diameter

$L_{\text {ref }} \quad$ Reference length

$L / D \quad$ Lift to drag ratio

$M \quad$ Mach number

$S_{\text {ref }} \quad$ Reference area

$T$ Temperature
U Velocity

Subscripts

()$_{\infty} \quad$ Freestream quantity

Symbols

$\alpha \quad$ Angle of attack

$\Delta \quad$ Change in quantity

$\rho \quad$ Density

* Research Engineer, Aerothermodynamics Branch, Senior Member AIAA

${ }^{\dagger}$ Research Engineer, Aerothermodynamics Branch, Member AIAA

${ }^{\ddagger}$ Research Scientist, Member AIAA

This material is declared a work of the U.S. Government and is not subject to copyright protection in the United States. 


\section{Introduction}

7 HE Orion Crew Exploration Vehicle (CEV) is being designed by NASA and its industry partners as part 1 of the Agency's program to send human explorers back to the moon and onward to Mars. ${ }^{1}$ The CEV will be launched using the new Ares crew launch vehicle, and consists of the primary Command Module (CM), Service Module, and Launch Abort System. The Orion CM is similar in shape to, but larger than the Apollo capsule. It is being designed to fly missions to the International Space Station as soon as 2014, and the moon by 2020 .

The Orion aerodynamic database ${ }^{2,3}$ is currently being developed by the CEV Aerosciences Program (CAP). This aerodynamic database will be the basis for the guidance, navigation, and control system for the $\mathrm{CEV}$ from the time the full vehicle separates from Ares until the CM re-enters the atmosphere and lands. It incorporates aerodynamic data primarily from computational analysis and wind tunnel testing. Uncertainties from turbulence modeling, grid resolution, and other physical modeling are combined to provide database uncertainties. At present, the only accounting for the aerodynamic effects of recession are in the form of a safety factor to cover all of the uncertainties that have not been directly accounted for. The aerodatabase is updated on a regular basis to incorporate additional analyses. The hypersonic portion of the aerodatabase relies primarily on computational analysis using the same tools used in this study. Future versions of the aerodatabase will incorporate the baseline computations from this study.

The change in aerodynamics due to the recession of the ablative heatshield as the command module re-enters the earth's atmosphere is currently accounted for as an added uncertainty in the CM aerodynamic database. This additional uncertainty is based on engineering judgment, not detailed analysis. The analysis presented here is an initial attempt to bound the aerodynamic effects in order to assess how best to handle recession effects within the Orion $\mathrm{CM}$ aerodatabase.

Recession of the heatshield is dependent on many factors that vary throughout the flight trajectory, including angle of attack, atmospheric properties, and velocity. The recession depth at each location is independent of the recession at other locations, and thus the recession process produces a shape that is not analytically defined. This leads to a deformed shape that can not be easily parameterized from the initial spherical shape. These factors make it difficult to determine a set of recession shapes that will provide an estimate of the maximum recession expected over a range of trajectories.

\section{A. Recessed Geometries}

A series of recessed geometries were developed in earlier recession analyses for the Orion CM using the engineering tool CBAERo coupled with a recession analysis. ${ }^{4-6}$ The TPS recession histories were computed at 232 discrete heat-shield locations using heating environments from a CBAERO anchored turbulent aerothermal database. Recession shapes were computed along various CM entry trajectories. CBAERo was then used to compute the change in trim angle of attack along the trajectories using the recessed shapes.

The current study is based on the geometries developed by Kinney ${ }^{5}$ using a lunar skip trajectory which targeted a hypersonic $L / D$ ratio of 0.3 and had a hypersonic trim angle around $160^{\circ}$. This analysis showed that the maximum recession was $1.35 \mathrm{in}$ and produces a maximum difference in pitching moment of 0.003 This translated into a change in vehicle trim angle of approximately $1.5^{\circ}$ at $M=2.4$.

\section{B. Current Study Overview}

The current study compares the aerodynamics for the baseline CEV CM configuration with the aerodynamics for several recessed geometry CM configurations. Four recessed shapes were chosen from Kinney's previous study, ${ }^{5}$ corresponding to trajectory points at the first and second dynamic pressure peaks, Mach 10.4, and the final point in the analysis at Mach 2.4. High-fidelity CAD models were developed for each of the selected trajectory points. The deformed heatshield surface was developed by applying the computed recessions in a direction normal to the heatshield surface, while the aft-body was held constant. Flow solutions were then computed using viscous computational fluid dynamics (CFD) codes DPLR and LAURA, and the inviscid CFD code FelisA. All four trajectory points were simulated with FELISA and DPLR, using both the baseline and the appropriate recessed geometry. Only two of the trajectory points were run with LAURA. Additionally, solutions at additional angles of attack on both baseline and recessed shapes were computed with FELISA in order to provide an understanding of the slope of the pitching moment curve for the recessed geometries. The results of the computations are used to determine how the change in vehicle shape due to recession affects the 
aerodynamic forces and moments and trim characteristics of the vehicle. Additionally, these computations with Euler and Navier-Stokes methodolgies provide a validation of the results from the engineering tool CBAERo.

\section{CEV Vehicle Configuration}

\section{A. Baseline CEV-CM Geometry}

The CXP-15000 geometry was used for the baseline analysis with all codes. The configuration is shown in Figure 1. The vehicle dimensions are given in Figure 1(a). The line between the heatshield and the aftbody is located at the point where the heatshield shoulder is tangent to the aftbody For the recessed geometries, recession is allowed only on the heatshield; the aftbody is not allowed to recess. The axis orientation with the conventions for positive forces, moments, and flow angles is shown in Figure 1(b). Note that the angle of attack is measured from the apex of the aftbody cone, such that an angle of attack of $180^{\circ}$ gives a stagnation point on the center of the heatshield.

The reference quantities for the CEV CM configuration, as used in this study, are summarized in Table 1. The center of gravity (c.g.) location from the CBAERo analysis was used here for the moment reference center $(m r c)$, except as otherwise noted.

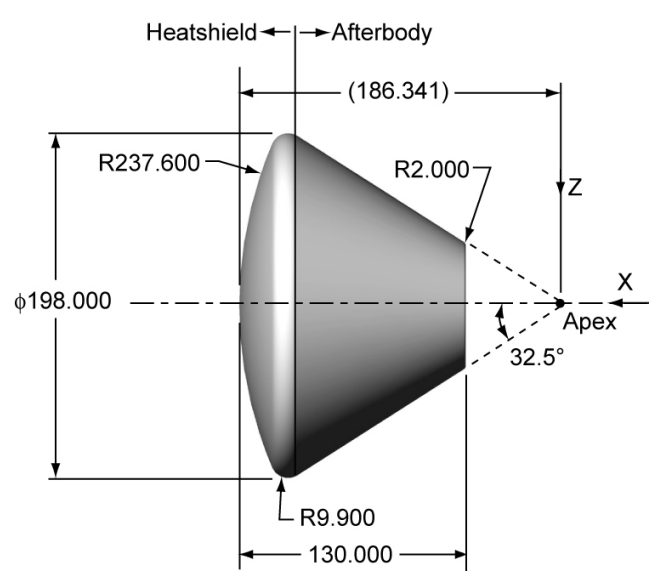

(a) Primary dimensions, in

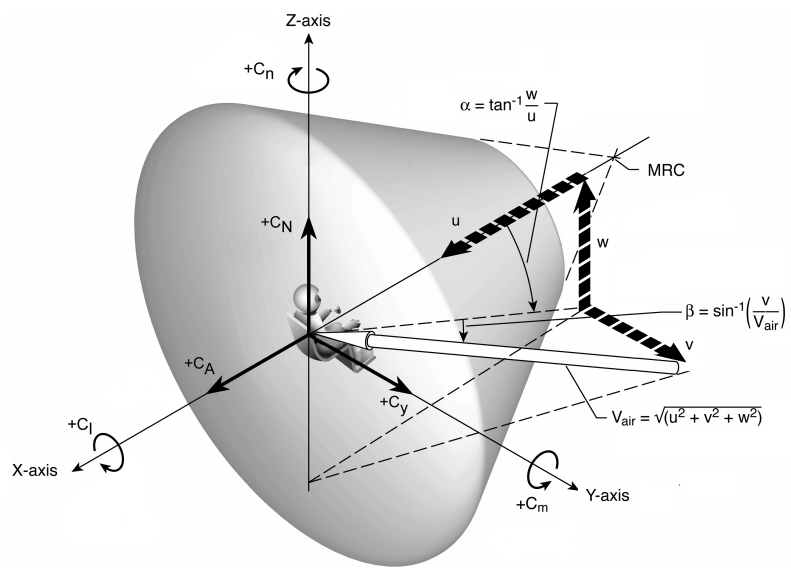

(b) Axis orientation for CEV CM

Figure 1. Dimensions of the CEV CM.

Table 1. Reference Quantities for CEV CM.

\begin{tabular}{ll} 
Reference Length, $L_{r e f}$ & $198 \mathrm{in}$ \\
Reference Area, $S_{r e f}$ & $30791 \mathrm{in}^{2}$ \\
Moment Reference Center, $m r c$ & $(134.32 \mathrm{in}, 0.0 \in,-7.031 \mathrm{in})$ \\
\hline${ }^{*}$ measured from vehicle apex &
\end{tabular}

\section{B. Recessed CEV-CM Geometries}

Four high-fidelity recessed CAD models were developed for this study, based on the discrete recessions computed by Kinney. ${ }^{5}$ The recessed geometries and the corresponding flow conditions for each case are given in Table 2. Note that the last geometry, 130, was developed for the final Mach 2.4 condition of the previous study. For this study, Mach 6 flow conditions are used with geometry 130.

In the recession analysis, only the heatshield surface was allowed to recess. The recession surface was blended to the aftbody cone to provide a smooth transition, with the body remaining constant from the location where the aftbody cone begins. Figure 2 shows the lower and upper shoulder regions for the final recessed geometry (130) with the outer mold line for the baseline and other recessed geometries overlaid. 
There is more recession on the lower shoulder than the upper. The differences between the baseline, 13, and 113 are noticeable in Figure 2. Subsequent geometries show a smaller progression of changes, and are difficult to visually distinguish at the scale shown in Figure 2. The maximum recession distance for each geometry is given in Table 2, and offers a quantitative comparison of the geometries.

Table 2. Recessed geometries from CBAERO analysis.

\begin{tabular}{clcccc} 
Geometry & Case Description & Mach & $\begin{array}{c}U_{\infty}, \\
\mathrm{km} / \mathrm{s}\end{array}$ & $\begin{array}{c}\alpha_{\text {trim }}, \\
\text { CBAERO }\end{array}$ & $\begin{array}{c}\text { Maximum } \\
\text { Recession, in }\end{array}$ \\
\hline 13 & 1st peak dynamic pressure & 28.7 & 9.0 & $160.1^{\circ}$ & 0.613 \\
113 & 2nd peak dynamic pressure & 17.0 & 5.6 & $158.9^{\circ}$ & 1.160 \\
120 & Mach 10 & 10.4 & 3.4 & $158.0^{\circ}$ & 1.310 \\
130 & Final Shape & 2.4 & 0.73 & $155.4^{\circ}$ & 1.345
\end{tabular}

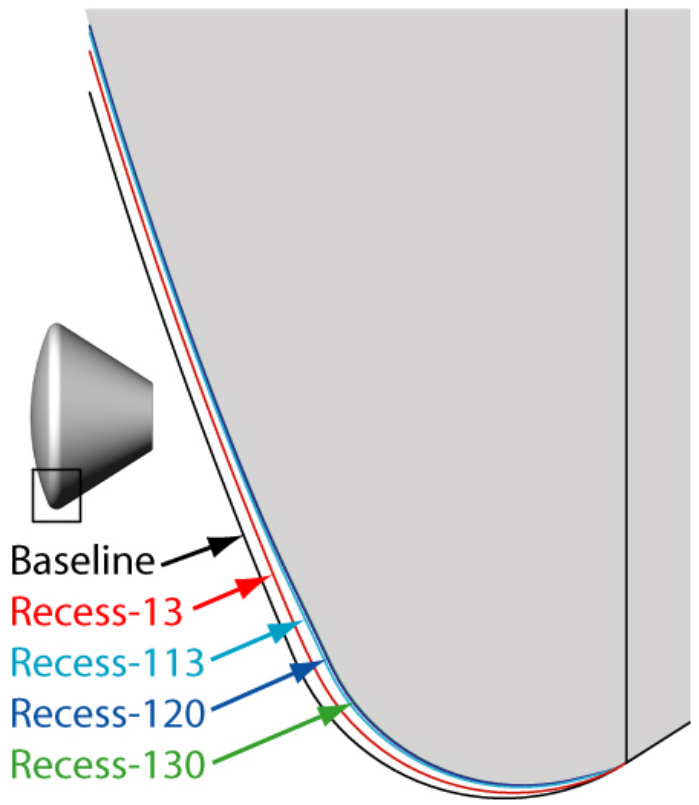

(a) Bottom / Windside.

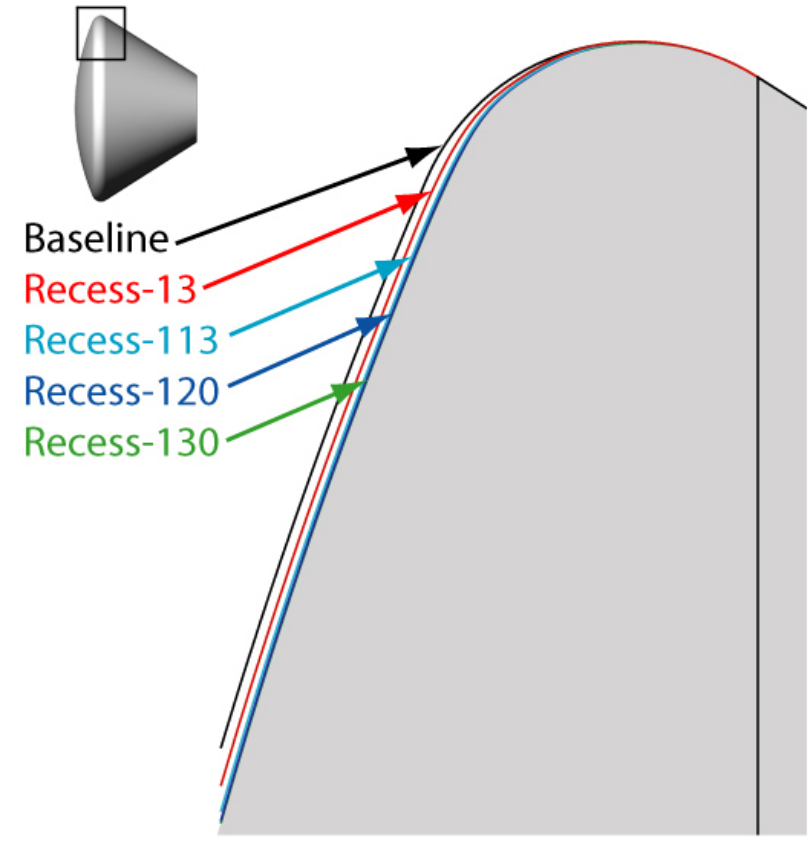

(b) Top /Leeside.

Figure 2. Comparison of baseline CEV geometry with recessed geometries.

\section{Methodology}

Three CFD tools were used in this study. The high-fidelity viscous solvers DPLR ${ }^{7}$ and LAURA ${ }^{8,9}$ were utilized at a single angle of attack for each condition. The unstructured, inviscid solver FELISA ${ }^{10,11}$ was used for angle of attack sweeps at each condition. The flow conditions and angle(s) of attack for each computation as well as the trim angle from the CBAERO analysis are given in Table 3. The conditions used to develop the recessed geometries were modified slightly for the current study. The angles of attack from the CBAERO trim analysis were adjusted for the present cases to match the usual aerodatabase angles of attack, $160^{\circ}$ for the higher Mach number cases, and $155^{\circ}$ for the Mach 6 case on the final recessed shape. This set of angles of attack is referred to as the primary angles of attack in the discussion of the study results. The velocities and temperatures were rounded to "nicer" numbers. For the Mach 6 case, the Mach number was fixed at 6.0 , and then the velocity was determined. Note that the geometry computed for a Mach 2.4 condition was 
analyzed at Mach 6 in this study ${ }^{\mathrm{a}}$.

Table 3. Flow conditions and angles of attack.

\begin{tabular}{ccccccccc} 
& & $U_{\infty}$, & $\rho_{\infty}$, & $T_{\infty}$, & $\alpha_{\text {trim }}$, & \multicolumn{3}{c}{$\alpha$ (for analysis) } \\
\cline { 7 - 9 } Geometry & Mach & $m / s$ & $k g / m^{3}$ & $K$ & CBAERO & DPLR & LAURA & FELISA $^{*}$ \\
\hline 13 & 28.7 & 9000 & $2.96 \mathrm{E}-04$ & 246.0 & $160.1^{\circ}$ & $160.0^{\circ}$ & - & $150.0^{\circ}-162.5^{\circ}$ \\
113 & 16.9 & 5500 & $6.99 \mathrm{E}-04$ & 265.5 & $158.9^{\circ}$ & $160.0^{\circ}$ & $160.0^{\circ}$ & $150.0^{\circ}-162.5^{\circ}$ \\
120 & 10.4 & 3400 & $1.58 \mathrm{E}-03$ & 268.5 & $158.0^{\circ}$ & $160.0^{\circ}$ & - & $150.0^{\circ}-162.5^{\circ}$ \\
130 & 6.0 & 1857 & $5.08 \mathrm{E}-03$ & 241.1 & $* *$ & $155.0^{\circ}$ & $155.0^{\circ}$ & $150.0^{\circ}-162.5^{\circ}$ \\
\hline${ }^{*} 150.0^{\circ}, 152.5^{\circ}, 155.0^{\circ}, 157.5^{\circ}, 160.0^{\circ}, 162.5^{\circ}$ \\
** Geometry corresponds to $M=2.4, \alpha_{\text {trim }}=155.4^{\circ}$ condition from CAP-AR-31. $\alpha_{\text {trim }}$ at $M=6$ not \\
provided.
\end{tabular}

\section{A. DPLR}

The meshes and solutions for the DPLR baseline cases were generated first. A previously explored grid resolution study ensured that the volume grids were sufficiently dense to capture the flow features around the vehicle and generate high quality aeroheating solutions. ${ }^{12}$ The surface mesh contains 28,512 points. The volume grid has 129 points in the normal direction, for a total of 3.7 million points.

Solutions were started and converged on either with the initial hyperbolic volume grid or a previously tailored volume grid. Grid adaptation was performed for each case using DPLR's internal grid adaptation algorithm that aligns the grid outer boundary with the bow shock formed around the vehicle, adjusts the grid cell spacing at the wall to maintain a cell Reynolds number of one, and interpolates the solution onto the newly adapted grid to speed convergence. The solution was then converged on the adapted grid. This process was repeated a minimum of three times for each case to ensure well-tailored grids that produce high quality solutions.

The tailored volume grids from the baseline solutions were used as a starting point to generate new grids for the recessed vehicle geometries. Surface grids for the recessed geometries were generated manually with GRIDGEN ${ }^{13}$ using the provided CAD databases, and merged with the non-recessed volume grids. The result for each case was a volume grid with the surface of the recessed geometry and the outer boundary of the adapted non-recessed case. The recessed geometry cases were started from the interpolated baseline solutions to speed convergence. For each DPLR case, both laminar and turbulent flow fields were computed.

The DPLR CFD solutions were generated using the DPLR flow solver version 3.05. ${ }^{12,14}$ DPLR solves the full set of Navier-Stokes equations and includes the effects of finite-rate chemistry and thermal nonequilibrium. The DPLR solver computes inviscid fluxes using a modified Steger-Warming flux splitting. Third-order accuracy is achieved using MUSCL extrapolation with a minmod flux limiter. Viscous fluxes are computed with second-order accurate central differencing. Transport properties are modeled with the Yos mixing rule. Diffusion coefficients are modeled using the Self Consistent Effective Binary Diffusion (SCEBD) model. A two-temperature model with the vibrational temperature in non-equilibrium and fully coupled finite rate chemistry modeled with Park's 1990 curve fits for five-species air was employed for all cases. A radiative equilibrium wall temperature boundary condition was used with a constant emissivity of 0.85. Surface recombination was modeled with a fully catalytic condition, defined as a boundary condition which forces the atomic species $(\mathrm{O}, \mathrm{N})$ to their diatomic state $\left(\mathrm{O}_{2}, \mathrm{~N}_{2}\right)$ at the wall . For turbulent cases, the flow is modeled as fully turbulent using Menter's two-equation shear stress transport (SST) turbulence model $^{15}$ with a compressibility correction. Turbulent values of Prandtl and Schmidt numbers of 0.9 and 0.7, respectively are used. Iterative convergence of the DPLR solutions is determined by monitoring differences over a set number of iterations in surface temperature, heat flux, and pressure coefficient, with convergence of the aerodynamic coefficients as a secondary check.

\footnotetext{
a The 130 geometry was needed for other studies by the CAP team, and the recession between Mach 6 and Mach 2.4 is negligible.

b For this condition, if NO strikes the wall it remains NO.
} 


\section{B. LAURA}

For the LAURA computations, the GRIDGEN ${ }^{13}$ package was used to generate a multi-block grid system for the original outer mold line of the CEV geometry based on the analytical definition of the CEV CM. High grid quality is maintained with grid lines from the surface to the outer boundary using elliptic partial differential equation smoothing with Steger and Sorenson source terms to control near wall orthogonality and grid line straightness through the volume to the outer flow domain. The initial grid system is generated with "inviscid" spacing, with respect to the normal direction, at the surface. The surface grid of the "inviscid" spaced volume grid is then projected onto each of the ablated surface CAD geometries. NASA's Volume Grid Manipulator ${ }^{16}$ software was then used to redistribute the points along the K-lines of the final projected volume grid to achieve viscous-type spacing for the boundary layer. Each grid system has total of 2.2 million cells in 40 equally dimensioned blocks.

The LAURA code ${ }^{8,9}$ is a CFD solver specialized for hypersonic re-entry physics and chemistry. LAURA has been validated against Orbiter laminar aerothermodynamic flight data. ${ }^{17}$ It is a three-dimensional upwind finite-volume solver with perfect gas, equilibrium, and finite-rate chemistry models. Point-relaxation is used to integrate in time to steady state. Roe-averaging ${ }^{18}$ with Harten's entropy fix ${ }^{19}$ and Yee's Symmetric Total Variation Diminishing limiter ${ }^{20}$ are used to formulate the inviscid fluxes while a second order scheme solves the viscous fluxes. Binary diffusion is employed with a variable Schmidt number.

The full Navier-Stokes formulation of the governing equations was used for the CEV flight simulations. ${ }^{21}$ The chemical kinetics for the Mach 16.9 flow fields were modeled with Park's 1993 kinetic model ${ }^{22}$ for fivespecies air in chemical and thermal non-equilibrium flow. As with the DPLR cases, the surface was assumed to be fully catalytic and in radiative equilibrium with an emissivity value of 0.85 . The laminar momentum and energy transport used the Wright ${ }^{23}$ curve fits for the collision integral coefficients. For the Mach 6 cases, a calorically perfect gas with a constant wall temperature of $500 \mathrm{~K}$ was used. Turbulent flow fields were computed using the Cebeci-Smith turbulence model. Iterative convergence was monitored in a similar manner as for the DPLR solutions.

\section{Felisa}

The baseline geometry used for the FELISA mesh generation was developed analytically, from the parameters (diameter, aft cone angle, heatshield radius, etc.) describing the CEV geometry. The recession shapes were provided as discrete CAD geometries. The discrete recessed heatshield was blended with the baseline geometry, keeping the curves that define the intersection of the aft cone with the heatshield corner as they were defined in the baseline geometry. It was verified that the aftbody of the recessed geometries exactly matched the baseline aftbody. This approach enssured that the aftbody definition stayed constant for all of the recessed geometries.

The meshing framework GRIDEx, ${ }^{24,25}$ with the FELISA meshing plugin was used for the unstructured mesh generation. The initial meshes used for baseline and recessed cases had similar spacing distributions, and comparable mesh sizes, with approximately $67 \mathrm{k}$ boundary triangles, $1.5 \mathrm{M}$ volume nodes, and $9.0 \mathrm{M}$ volume tetrahedra.

The FeLisa flow solver models the inviscid Euler equations with the Hänel flux vector splitting formulation $^{26}$ on unstructured, tetrahedral meshes. All FELISA simulations were performed with an equilibrium air gas model, based on McBride's ${ }^{27}$ curve fits for the thermodynamic properties of air in equilibrium. Iterative convergence of the flow solution was determined by convergence of the primary aerodynamic forces as well as the maximum and minimum total enthalpy. The baseline solutions were adapted using a solution gradient based approach. ${ }^{28,29}$ Adapted meshes ranged in size from 1.7M to $4.1 \mathrm{M}$ volume nodes. Mesh size increased with successive adaptations, with increasing Mach number, and with the recessed geometries as compared to the baseline.

\section{Limitations, Assumptions, and Constraints}

The limitations of this study are primarily related to limitations of the provided recessed geometries and to the general limitations and assumptions of the methodologies used. A brief discussion of the limitations and assumptions in this analysis is included here, to allow for tempered interpretation of the study results.

The provided geometries do not bound the range of recession shapes that could be encountered in flight, such that no general conclusions about limits of expected effects due to recession can be made. In particular, the shape and depth of recessed geometry could be quite different from shapes analyzed. The recession 
analysis was based on a particular trajectory tailored for a hypersonic $L / D$ ratio of 0.3 , and corrsponding trim angle of attack of $\alpha_{\text {trim }} \sim 160^{\circ}$. This angle of attack places the stagnation region well ahead of the shoulder region, such that the highest heating rates are in an area of low curvature on the heatshield. For trajectories which target a higher $L / D$, up to 0.4 , the trim angle is lower, and therefore the stagnation region is closer to the shoulder where there is a smaller radius of curvature. This orientation will result in higher heating in the stagnation region, leading to more recession at the shoulder. In fact, the initial CBAERo study ${ }^{4}$ shows this, and predicts a larger change in trim angle (up to $2^{\circ}$ ) than the geometries used in the current study. Nothing in this analysis accounts for the change in aerodynamics if the location of the maximum recession shifts, producing a more (or less) asymmetric shape. These geometries do provide worst case recession for this particular series of trajectories with this specific c.g. location, as they are based on the lunar skip trajectory which produces the greatest recession of the various lunar and low earth orbit trajectories.

This analysis computes the moment coefficients about a constant $m r c$ location. In a full flight simulation, the aerodatabase is responsible for providing aerodynamic coefficients with moments about a single $m r c$, and the trajectory model is responsible for providing the c.g. location as a function of the trajectory. The trim angle, $\alpha_{\text {trim }}$, is thus a function of the aerodatabase and the trajectory (flight conditions and shifting c.g. location). In this study, we look at the change in $\alpha_{\text {trim }}$ between the baseline and recessed geometries in order to quantify the recession effect. These results must be interpreted in the context of a constant c.g., with the recognition that the shift in trim angle over a complete trajectory is not completely modeled.

Because the CFD models are not coupled with ablation analysis, this analysis does not account for potential aerodynamic increments due to the ablation process itself. This process produces a blowing from the surface that dramatically changes the character of the boundary layer and reduces the heating rates. The ablation process could have an effect on the aerodynamics in addition to the heating.

The typical limitations and assumptions associated with CFD modeling of the aerodynamics apply to this analysis as well. The inviscid solver used (FELISA) employs an equilibrium chemistry model, which misses the non-equilibrium effects that can be significant above Mach 20. The aftbody flowfields for baseline and recessed geometries have not been fully resolved, either for the inviscid or viscous meshes. There has been no validation of the turbulence models for the highly separated aft-body flow. However, the impact of the lack of validation is offset by the small magnitude of the aerodynamic loads in this region as compared to the loads on the heatshield.

\section{Results and Discussion}

The analyses of the baseline and recessed geometry aerodynamics are grouped into four sections. First, an overview of the the aerodynamic forces and moments is given by examining a series of plots of the forces and moments vs. the angle of attack at each of the flow conditions. Trends in the differences between the baseline and recessed forces and moments are established, and related to the Orion CM aerodynamic database uncertainties. The second section looks more closely at the aerodynamics as a function of decreasing Mach number and thus increasing recession, for the primary angle of attack at each flow condition. Differences between the various computational methodologies are examined and the trend in the change in pitching moment with increasing recession is more clearly established. The third section uses the pitching moments and $L / D$ ratios to examine how the vehicle trim angle and the $L / D$ ratio at trim change with increasing recession, and compares these results with the earlier Kinney study. ${ }^{5}$ The final section looks at how a (constant) shift in the vehicle's center of gravity affects the change in trim angle due to recession. The results presented here provide an initial quantification of the aerodynamic effects of recession, and suggest possible methods of incorporating the results into the Orion CM hypersonic database.

\section{A. Overview of Forces and Moments}

The aerodynamic forces and moments for the DPLR turbulent and FELISA computations are given in this section to provide an overview of how the vehicle aerodynamics change with recession. The results for the DPLR laminar and the LAURA computations are similar to the DPLR turbulent computations, and will be examined in more detail in the following section. The force data for $U_{\infty}=3.4 \mathrm{~km} / \mathrm{s}(M=10.4)$ and $U_{\infty}=$ $5.5 \mathrm{~km} / \mathrm{s}(M=16.9)$ are not included in this section to simplify the discussion; it is very similar to the $U_{\infty}$ $=1.9 \mathrm{~km} / \mathrm{s}(M=6)$ data. 


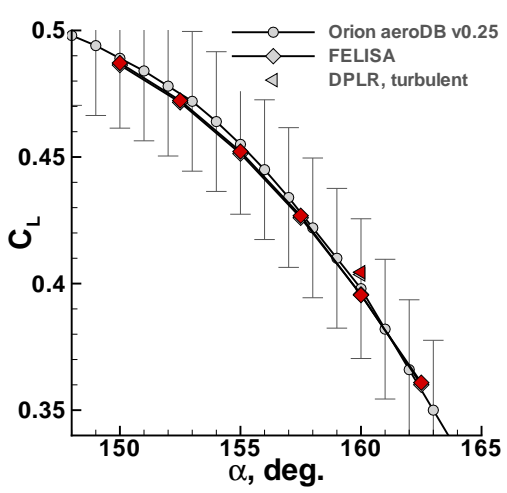

(a) $U_{\infty}=9 \mathrm{~km} / \mathrm{s}, M=28.7$

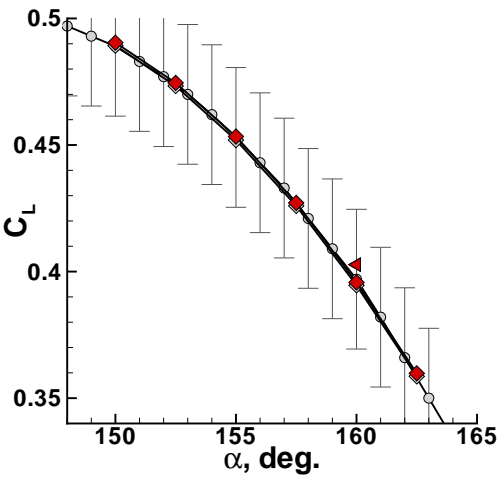

(b) $U_{\infty}=5.5 \mathrm{~km} / \mathrm{s}, M=16.9$

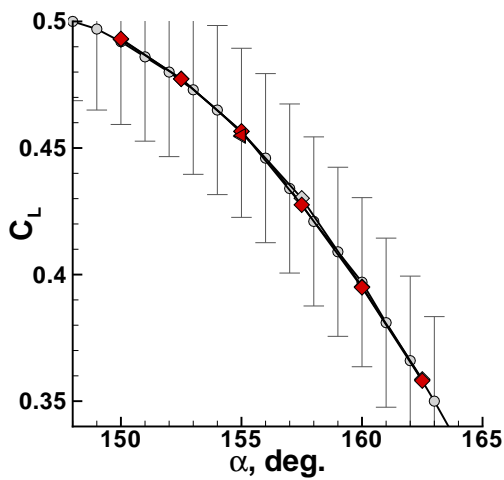

(c) $U_{\infty}=1.9 \mathrm{~km} / \mathrm{s}, M=6$

Figure 3. Comparison of lift coefficient for baseline and recessed geometries, over range of angles of attack. Grey symbols are baseline geometry, filled red symbols are recessed geometry.

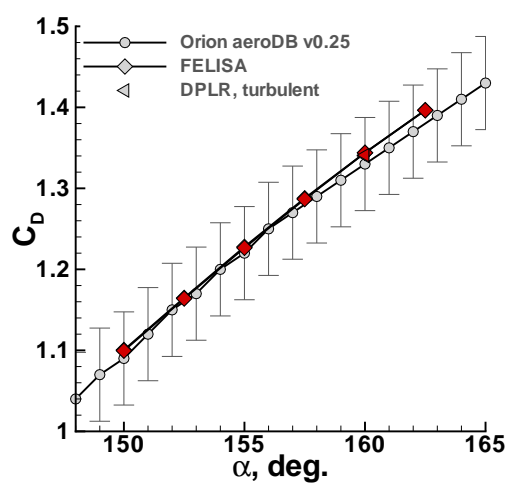

(a) $U_{\infty}=9 \mathrm{~km} / \mathrm{s}, M=28.7$

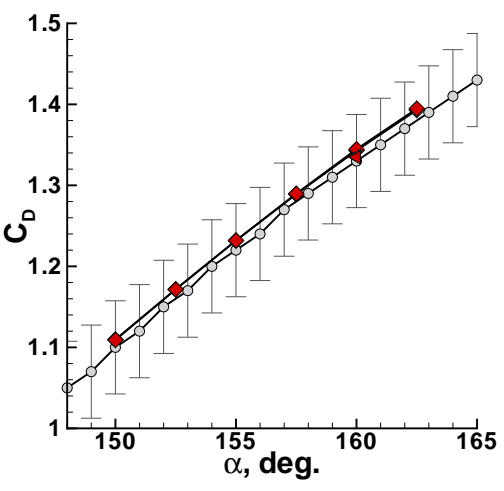

(b) $U_{\infty}=5.5 \mathrm{~km} / \mathrm{s}, M=16.9$

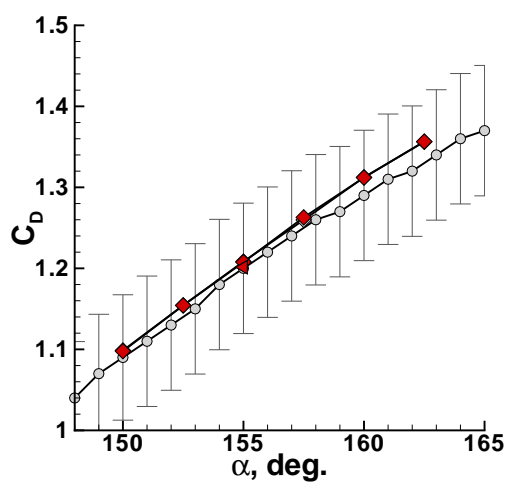

(c) $U_{\infty}=1.9 \mathrm{~km} / \mathrm{s}, M=6$

Figure 4. Comparison of drag coefficient for baseline and recessed geometries, over range of angles of attack. Grey symbols are baseline geometry, filled red symbols are recessed geometry.

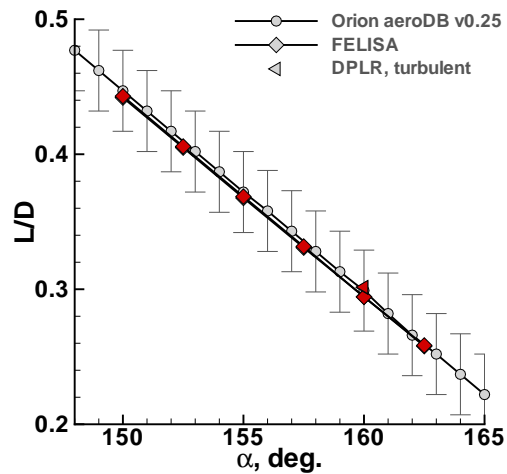

(a) $U_{\infty}=9 \mathrm{~km} / \mathrm{s}, M=28.7$

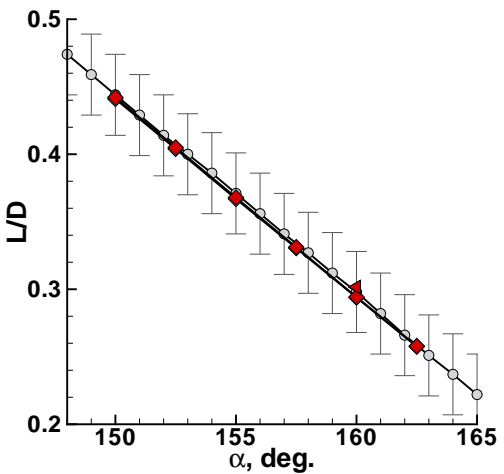

(b) $U_{\infty}=5.5 \mathrm{~km} / \mathrm{s}, M=16.9$

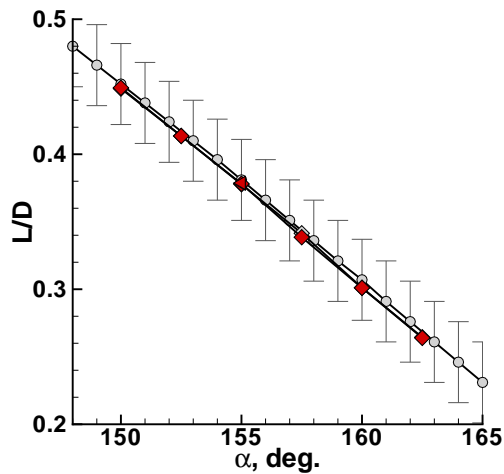

(c) $U_{\infty}=1.9 \mathrm{~km} / \mathrm{s}, M=6$

Figure 5. Comparison of $L / D$ for baseline and recessed geometries, over range of angles of attack. Grey symbols are baseline geometry, filled red symbols are recessed geometry.

Figures 3 through 6 show the aerodynamic forces and moments for the baseline and recessed geometry cases compared to the Orion CM aerodynamic database, version $0.25,{ }^{2}$ with baseline geometry uncertainties. For each flow condition, the aerodynamic data are presented as $C_{L}, C_{D}, L / D$, and $C_{m}$ plotted against angle of attack. The grey symbols on each plot represent the baseline geometry, and the red symbols show the 
recessed geometry. For each plot, there is only one set of DPLR results (at the primary angle of attack).

The forces show small differences between baseline and recessed geometries, with the differences indistinguishable at the scale of the plots. The differences between the methodologies and the database values are small, and well within the database uncertainty.

Figure 6 shows the effect of recession on the pitching moment. The change in the pitching moment due to the recession is small for the highest velocity case and increases as the velocity decreases. The values of the pitching moment remain within the uncertainty bounds for $C_{m}$ over the range of velocities and angles of attack. The FELISA results show that, for each flow condition, $\Delta C_{m}$ is approximately constant across the angle of attack range, and that the slope of the curve is approximately the same as for the aerodatabase. Note that the slopes of the $L / D$ vs angle of attack curves are also the same. The slopes of these curves will be used later in determining the trim angle of attack and the $L / D$ at the new trim angle.

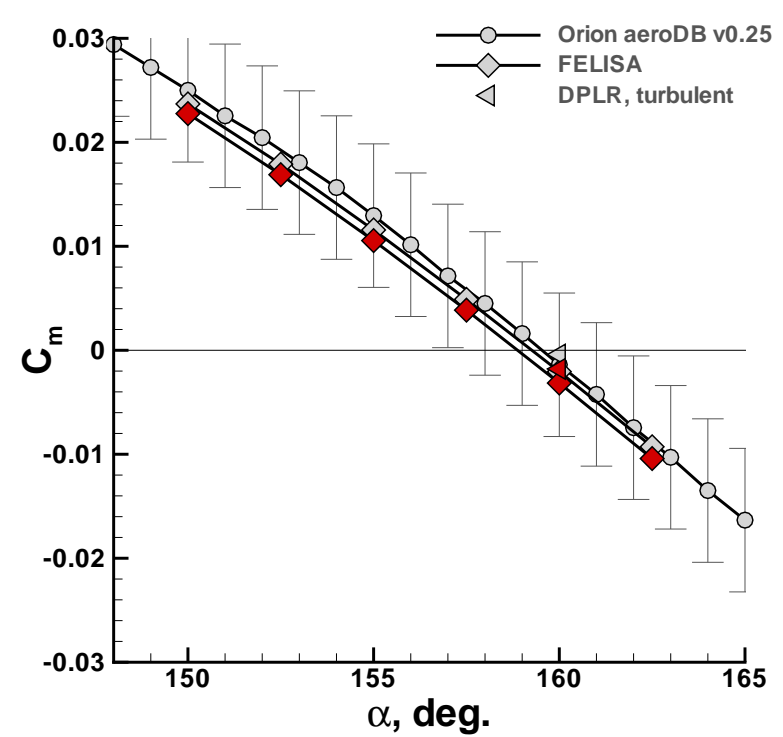

(a) $U_{\infty}=9 \mathrm{~km} / \mathrm{s}, M=28.7$

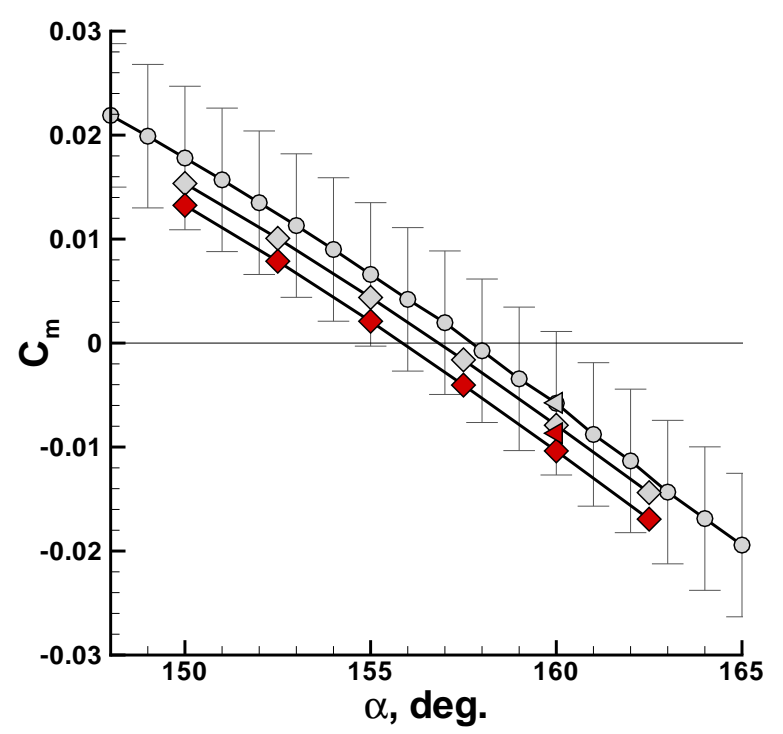

(c) $U_{\infty}=3.4 \mathrm{~km} / \mathrm{s}, M=10.4$

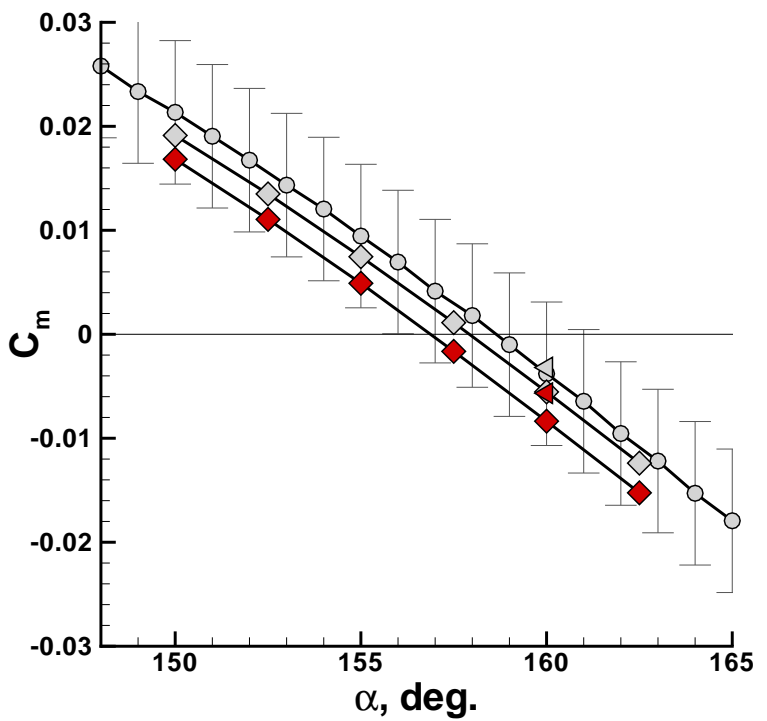

(b) $U_{\infty}=5.5 \mathrm{~km} / \mathrm{s}, M=16.9$

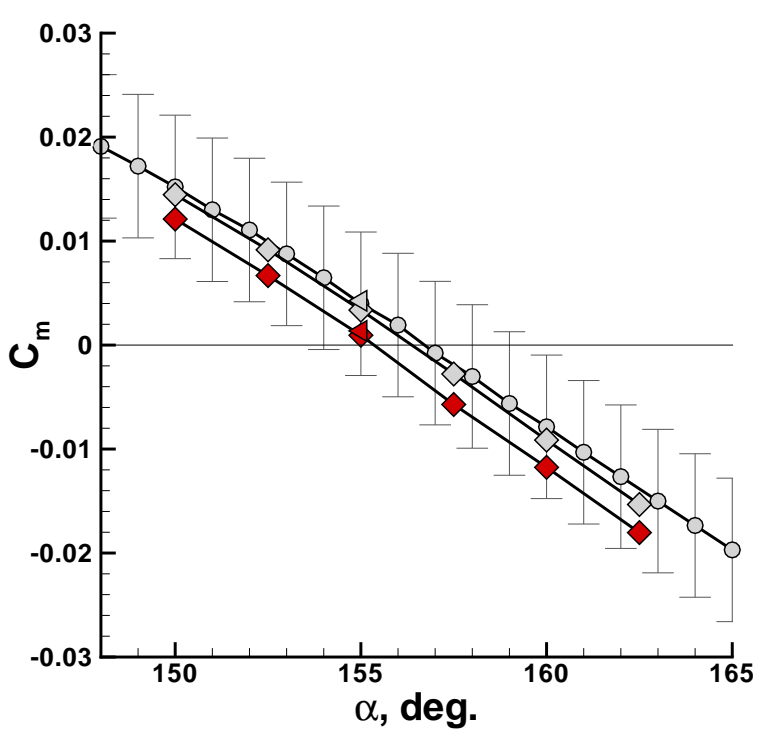

(d) $U_{\infty}=1.9 \mathrm{~km} / \mathrm{s}, M=6$

Figure 6. Comparison of pitching moment coefficient for baseline and recessed geometries, over range of angles of attack. Grey symbols are baseline geometry, filled red symbols are recessed geometry. 


\section{B. Aerodynamic Forces and Moments at primary analysis conditions}

This section focuses on the comparison of the aerodynamic forces and moments for all of the computations at the primary angle of attack for each flow condition. Differences between the various codes and between laminar and turbulent computations are examined. The effect of the recession on the aerodynamics is quantified for the range of conditions.

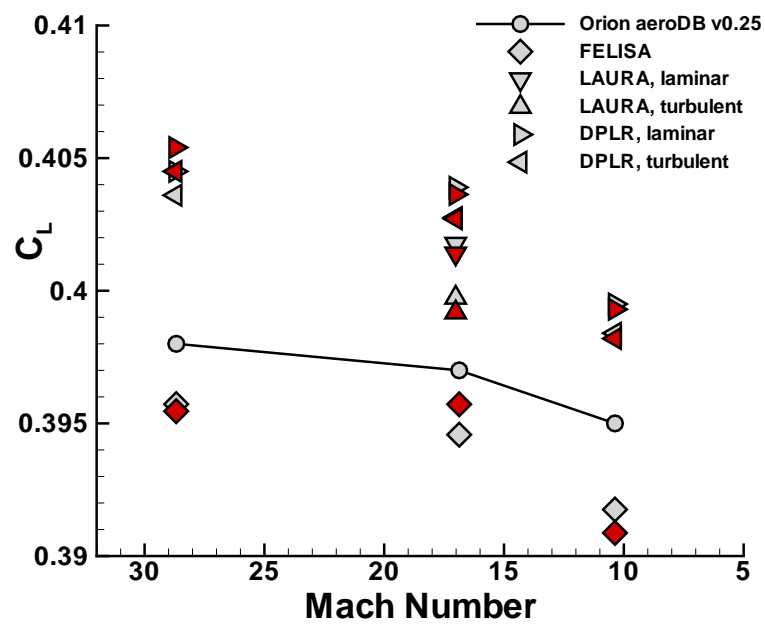

(a) $C_{L}$

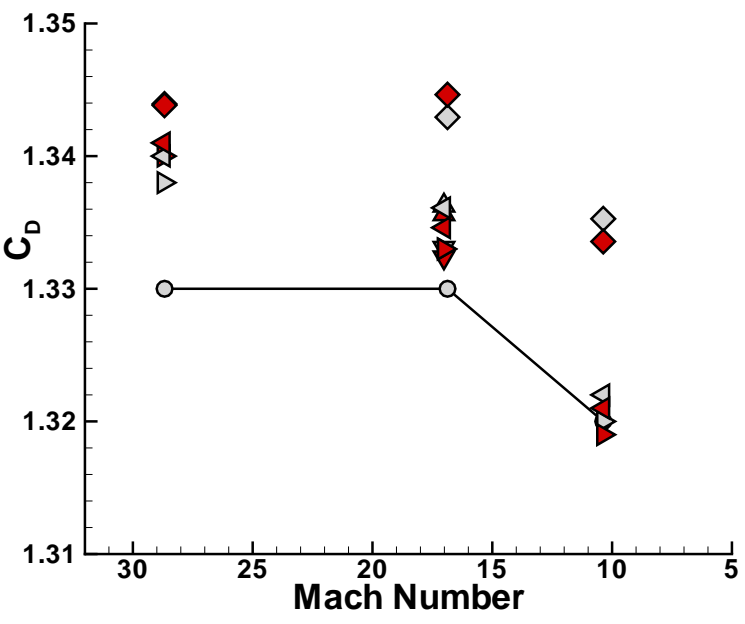

(b) $C_{D}$

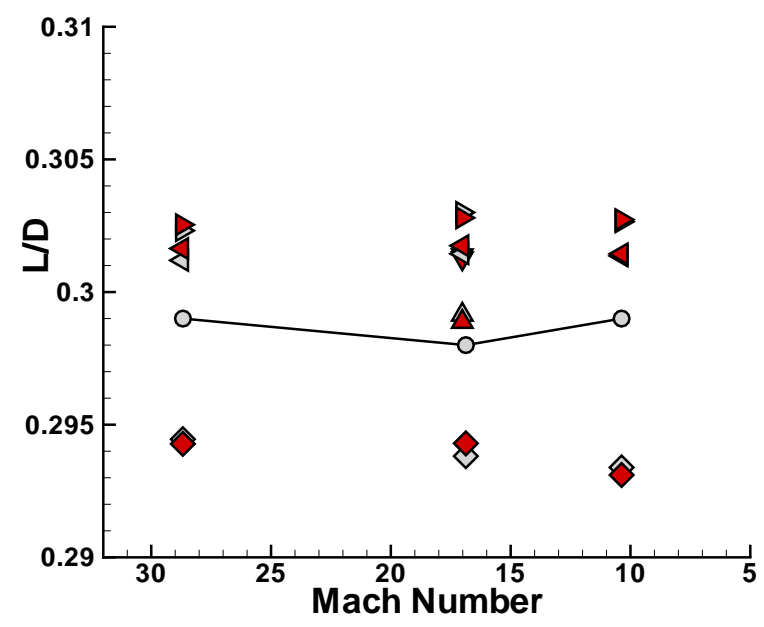

(c) $L / D$

Figure 7. Comparison of $C_{L}, C_{D}$, and $L / D$ for baseline and recessed geometries, over the Mach number range, at $160^{\circ}$. Grey symbols are baseline geometry, filled red symbols are recessed geometry.

Figure 7 shows the lift and drag coefficients and the lift to drag ratio for all of the computations at $160^{\circ}$ plotted against decreasing Mach number. Note that the recession increases from left to right. The scales of the coefficient axes are greatly expanded from the scale of the previous plots, to allow for more detailed comparison. The data from the Orion CM aerodatabase is included for comparison as in the previous section, but the uncertainties are omitted as the range of the force coefficients is an order of magnitude smaller than the uncertainty bounds. Each pair of baseline and recessed solutions computed with the same solver and turbulence model has a different symbol. As in the Figures 3-5, the grey symbols represent baseline computations, and the red filled symbols represent the recessed geometry computations.

There is little difference between the baseline and recessed $C_{L}$ and $L / D$ ratios computed with the same methodology, as seen by the nearly overplotted gray and red symbols. The difference in $C_{D}$ is more evident, but is still very small. There is a small but noticeable variation between laminar and turbulent solutions computed with the same solver. A greater variation occurs between the different solvers, with the aero- 
database value usually between the inviscid FELISA results and the higher fidelity DPLR and LAURA results. Recalling the database uncertainties as plotted in Figures 3-5, and noting the magnified scale of these plots, it is clear that the variations in $C_{L}, C_{D}$, and $L / D$ among all of the methodologies and geometries are well within the uncertainties of the database.

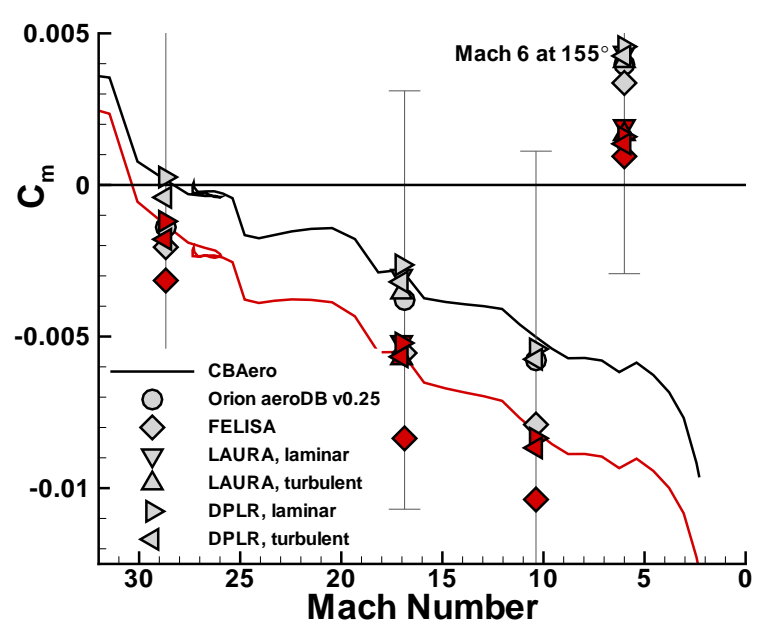

(a) $C_{m}$

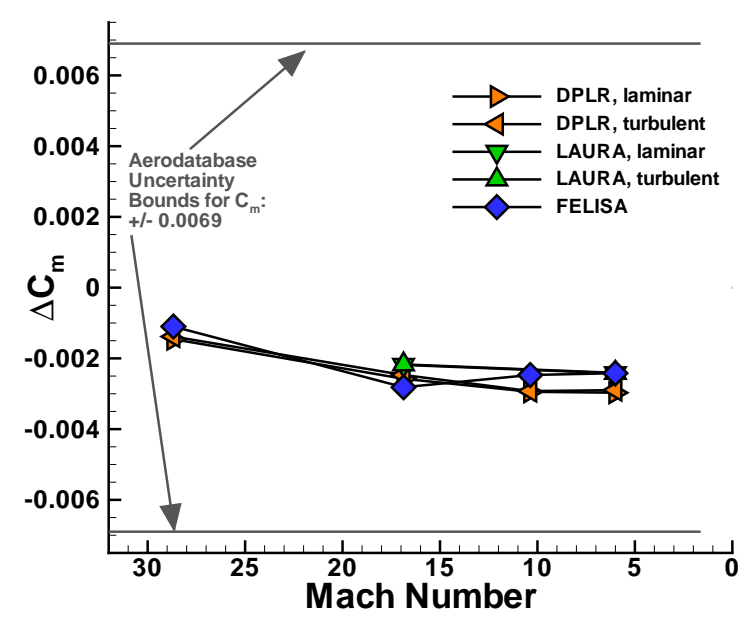

(b) $\Delta C_{m}$

Figure 8. Comparison of $C_{m}$ and $\Delta C_{m}$ for baseline and recessed geometries, for all primary angle of attack computations.

The pitching moments for all computations are given in Figure 8 as a function of decreasing Mach number. Figure 8(a) follows the format of the previous figure, with the addition of the Mach 6 computations at $155^{\circ}$ and uncertainty bounds on the aerodatabase moments. The pitching moments from the CBAERO analysis ${ }^{5}$ at a constant angle of attack of $160^{\circ}$ are also included in this plot. Figure $8(\mathrm{~b})$ shows the difference between the pitching moments for the baseline and recessed geometries, computed as $\Delta C_{m}=C_{m_{\text {recess }}}-C_{m_{\text {baseline }}}$. The average values for $\Delta C_{m}$ at each conditions are given in Table 4 . The changes $C_{L}, C_{D}$, and $L / D$ are included for completeness, and are computed in the same way as $\Delta C_{m}$.

Table 4. Average change in aerodynamic quantities for each condition.

\begin{tabular}{ccccc} 
Mach & $\Delta C_{L}$ & $\Delta C_{D}$ & $\Delta(L / D)$ & $\Delta C_{m}$ \\
\hline 28.68 & 0.0005 & 0.0010 & 0.0002 & -0.0013 \\
16.87 & -0.0000 & -0.0002 & 0.0000 & -0.0024 \\
10.37 & -0.0004 & -0.0012 & 0.0000 & -0.0028 \\
6.00 & 0.0001 & -0.0001 & 0.0001 & -0.0026
\end{tabular}

The pitching moments, unlike the forces, show a clear difference between the baseline and recessed geometries. The change in the pitching moment due to the recession is small for the highest velocity case, with $\Delta C_{m}$ between -0.0011 and -0.0015 . For the $U_{\infty}=5.5 \mathrm{~km} / \mathrm{s}$ condition, the differences in the pitching moments between baseline and recessed increase to approximately twice that calculated for the $U_{\infty}=9 \mathrm{~km} / \mathrm{s}$ condition, with $\Delta C_{m}$ between -0.0022 and -0.0028 , and an average of -0.0024 . As recession increases, the difference in pitching moment levels off, with the average $\Delta C_{m}=-0.0026$ at the final condition. The $\Delta C_{m}$ values computed here could be used as a starting point to incorporate recession effects into the Orion aerodynamic database, whether as an added uncertainty or an increment on the pitching moment.

Figure 8(a) also gives a clearer picture of the variations in $C_{m}$ for the different methodologies employed in the study. The difference in $C_{m}$ between laminar and turbulent solutions is smaller than $\Delta C_{m}$. The DPLR and LAURA solutions are very similar, and are closer to the aerodatabase value than FELISA for all cases except the highest Mach number case. The pitching moment values for the FELISA solutions are consistently lower than for the DPLR and LAURA solutions. The results from the CBAERo analysis track well with the 
current results, and suggest that an engineering approach to quantifying recession effects will be adequate for further quantifying recession effects, although occasional checks with the higher-fidelity tools would be advisable.

\section{Trim angle trends}

In order to assess the impact of the recession on stability and guidance, the trim angles of attack for each flow condition, with each solver, have been computed. From the perspective of maintaining stability on re-entry while the heatshield is ablating, the change in trim angle due to ablation is the important metric. The resulting change in $L / D$ at trim due to the change in trim angle is important to ensure sufficient cross-range capability and landing precision.

The computation of the trim angle $\alpha_{\text {trim }}$ and change in $L / D$ due to the shift in $\alpha_{\text {trim }}$ is performed consistently for each flow solution. The slopes of the $C_{m}$ vs. $\alpha$ curve from the aerodatabase is determined for each flow condition, and used to extrapolate $\alpha_{\text {trim }}$ from the $C_{m}$ value computed at the primary angle of attack. This approach assumes that (1) the variation of $C_{m}$ with respect to $\alpha$ is linear between the primary angle of attack and the trim region and that (2) the slope of the $C_{m}$ vs. $\alpha$ curve is the same for the baseline and recessed geometries. These assumptions are supported by the FELISA angle of attack sweep computations, as shown in the first section. Note that the angle of attack sweep data from the FeLISA computations is not used to compute the slope of the $C_{m}$ vs. $\alpha$ curve, in order to maintain a consistent comparison with the DPLR and LAURA results. The computation for $L / D$ at $\alpha_{\text {trim }}$ is similar. The slope of the $L / D$ vs. $\alpha$ curve from the aerodatabase is used with the computed $\alpha_{\text {trim }}$ to extrapolate $(L / D)_{\text {trim }}$ for each case. Again, the FeLisa results show that the slope of the $L / D$ vs. $\alpha$ curve does not change between the recessed and baseline geometries.

The changes in trim angle, $\Delta \alpha_{\text {trim }}$, and $L / D$ due the change in trim angle, $\Delta(L / D)_{\text {trim }}$, for all FeLISA, LAURA, and DPLR computations are plotted vs. decreasing Mach number in Figure 9. The CBAERo data from Kinney ${ }^{5}$ is included for comparison. The $\Delta \alpha_{\text {trim }}$ and $\Delta(L / D)_{\text {trim }}$ data is also tabulated in Table 5. Only the turbulent data points are included in the table for simplicity.

In general, the higher fidelity data follow the trend of the CBAERo analysis well, with the DPLR data consistently closest to the CBAERO data. The FELISA and LAURA data consistently predict a smaller $\Delta \alpha_{\text {trim }}$ and $\Delta(L / D)_{\text {trim }}$, with the exception of FELISA at the $U_{\infty}=5.5 \mathrm{~km} / \mathrm{s}(M=16.9)$ condition. There is very little difference between the laminar and turbulent data, as compared to the scatter between codes. The range of the dispersions among the high fidelity computations gives a $2 \sigma$ uncertainty estimate ${ }^{\mathrm{c}}$ in $\Delta \alpha_{\text {trim }}$ between $0.17^{\circ}$ and $0.24^{\circ}$, with an RMS average of $0.22^{\circ}$ over all conditions. For $\Delta(L / D)_{\text {trim }}$, the $2 \sigma$ uncertainty varies between 0.0035 and 0.0047 , with an RMS average of 0.0042 . The consistent trend of the CBAERO data with the high fidelity data suggests that the CBAERo computations provide reasonable estimates of the effects of recession on the trim angle and $L / D$.

Table 5. Summary of $\Delta \alpha_{\text {trim }}$ and $\Delta(L / D)_{t r i m}$, reference c.g. location

\begin{tabular}{|c|c|c|c|c|c|c|}
\hline \multirow[b]{2}{*}{ Mach } & \multicolumn{3}{|c|}{$\Delta \alpha_{\text {trim }}$} & \multicolumn{3}{|c|}{$\Delta(L / D)_{\text {trim }}$} \\
\hline & FELISA & LAURA (turb) & DPLR (turb) & FELisa & LAURA (turb) & DPLR (turb) \\
\hline 28.7 & $-0.43^{\circ}$ & - & $-0.54^{\circ}$ & 0.006 & - & 0.008 \\
\hline 16.9 & $-1.17^{\circ}$ & $-0.90^{\circ}$ & $-1.02^{\circ}$ & 0.018 & 0.013 & 0.015 \\
\hline 10.4 & $-1.08^{\circ}$ & - & $-1.27^{\circ}$ & 0.015 & - & 0.019 \\
\hline 6.0 & $-1.09^{\circ}$ & $-1.08^{\circ}$ & $-1.30^{\circ}$ & 0.016 & 0.015 & 0.019 \\
\hline
\end{tabular}

\section{Effect of Moment Reference Center on Trim Angle Change}

The previous sections discussed $\Delta \alpha_{\text {trim }}$ and $\Delta(L / D)_{\text {trim }}$ for a center of gravity location that provides for a nominal hypersonic flight $L / D$ of 0.3 . The target hypersonic $L / D$ ratio, and thus the nominal center of

\footnotetext{
${ }^{\mathrm{c}} \sigma=\bar{R} / d_{2}, d_{2}=1.693$ for $N=3, d_{2}=2.326$ for $N=5$.
} 


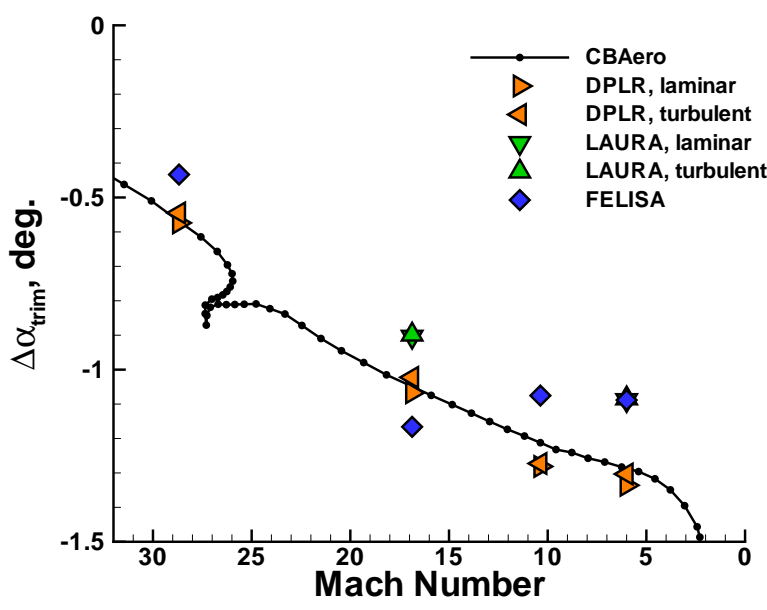

(a) $\Delta \alpha_{\text {trim }}$

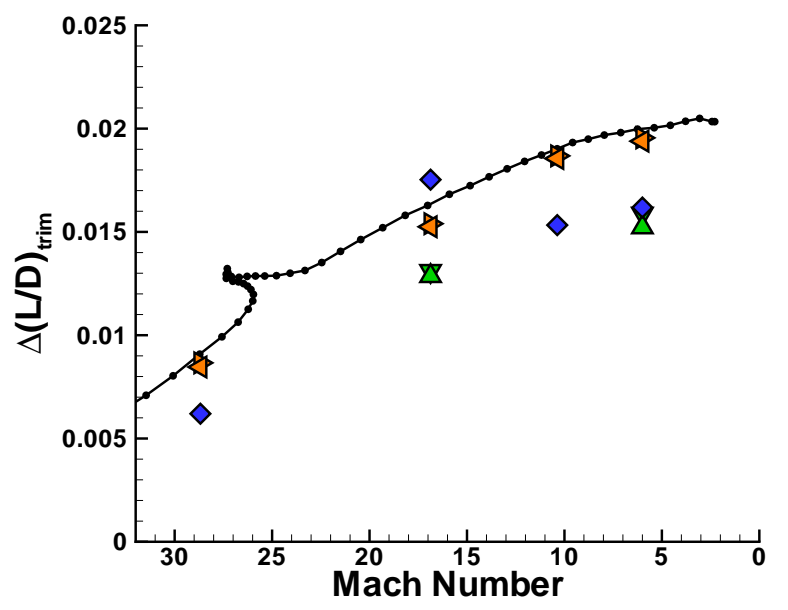

(b) $\Delta(L / D)_{\text {trim }}$

Figure 9. $\Delta \alpha_{\text {trim }}$ and $\Delta(L / D)_{\text {trim }}$ for all cases compared to CBAERo results.

gravity location, has shifted as the design and flight profile for the CEV has been refined, and will continue to shift throughout the design process.

Figure 10 repeats the format of Figure 6 , for the $U_{\infty}=9 \mathrm{~km} / \mathrm{s}$ and $U_{\infty}=1.9 \mathrm{~km} / \mathrm{s}$ cases, with the $m r c$ location shifted such that the hypersonic trim $L / D$ is approximately 0.4 , to (133.848in, $0.0 \mathrm{in},-9.504 \mathrm{in})$. These plots show a small change in slope from the $C_{m}$ vs. $\alpha$ plots of Figure 6 , and the expected shift of the trim angle to a lower angle of attack. Table 6 summarizes the changes in trim angle and $L / D$ at trim for the alternate $m r c$ location. Overall, $\Delta \alpha_{\text {trim }}$ values are about $10 \%$ lower for the alternate $m r c$ as compared to the original location. The $\Delta(L / D)_{\text {trim }}$ values are also lower, again on the order of $10 \%$. Therefore, as the nominal trim angle moves more toward the vehicle shoulder, the change in trim angle due to recession is somewhat lessened.

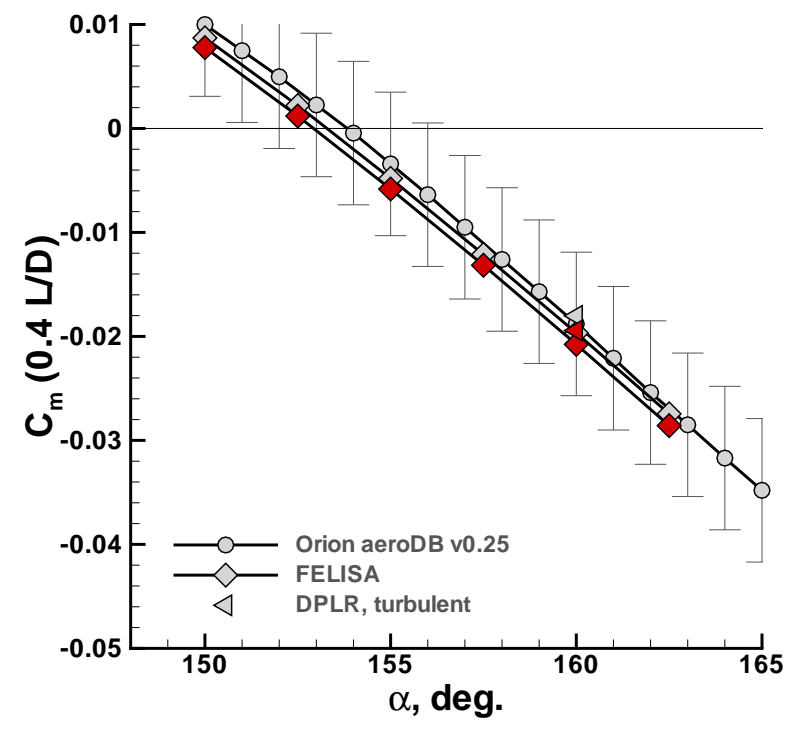

(a) $U_{\infty}=9 \mathrm{~km} / \mathrm{s}, M=28.7$

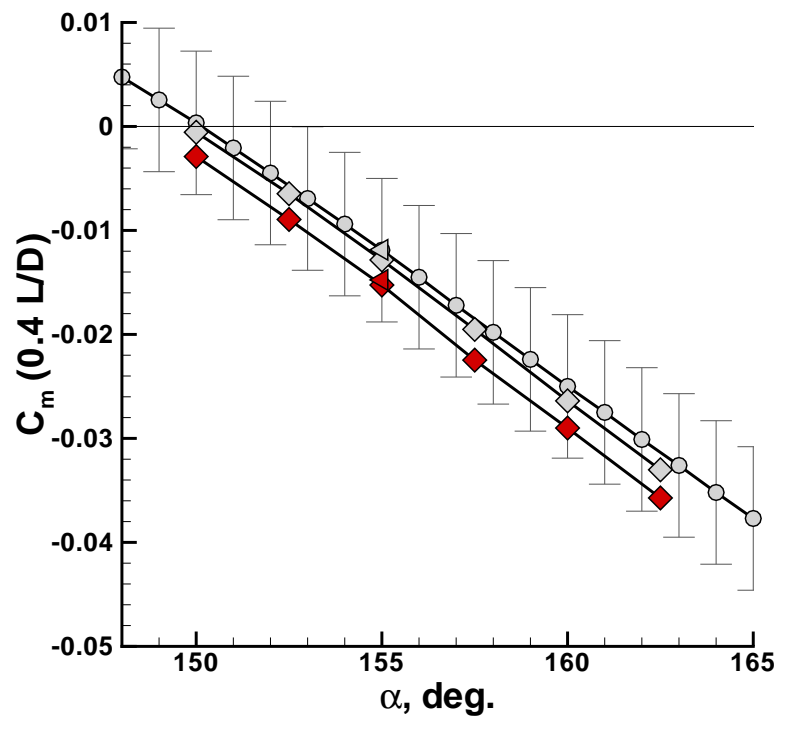

(b) $U_{\infty}=1.9 \mathrm{~km} / \mathrm{s}, M=6$

Figure 10. Comparison of $C_{m_{c g}}$ for baseline and recessed geometries. 
Table 6. Summary of $\Delta \alpha_{\text {trim }}$ and $\Delta(L / D)_{\text {trim }}$ for all cases, alternate c.g. location

\begin{tabular}{|c|c|c|c|c|c|c|}
\hline \multirow[b]{2}{*}{ Mach } & \multicolumn{3}{|c|}{$\Delta \alpha_{t r i m}$} & \multicolumn{3}{|c|}{$\Delta(L / D)_{\text {trim }}$} \\
\hline & FELISA & LAURA (turb) & DPLR (turb) & FELISA & LAURA (turb) & DPLR (turb) \\
\hline 28.7 & $-0.39^{\circ}$ & - & $-0.50^{\circ}$ & 0.006 & - & 0.008 \\
\hline 16.9 & $-1.07^{\circ}$ & $-0.81^{\circ}$ & $-0.92^{\circ}$ & 0.016 & 0.012 & 0.014 \\
\hline 10.4 & $-0.97^{\circ}$ & - & $-1.15^{\circ}$ & 0.014 & - & 0.017 \\
\hline 6.0 & $-0.99^{\circ}$ & $-0.99^{\circ}$ & $-1.19^{\circ}$ & 0.015 & 0.014 & 0.018 \\
\hline
\end{tabular}

\section{Concluding Remarks}

Recession of the heatshield due to ablation primarily affects the pitching moment and thus the trim angle of attack; the effects on $C_{L}, C_{D}$, and $L / D$ are negligible. Generally, moments show consistent differences between baseline and recessed geometries that correlate with the maximum amount of recession. The difference in pitching moment (between baseline and recessed) increases as the recession increases and the Mach number decreases. The change in trim angle of attack predicted by the analysis tools used here increases from $\sim 0.5^{\circ}$ at $U_{\infty}=9 \mathrm{~km} / \mathrm{s}$ to $\sim 1.3^{\circ}$ at $M=6$, and is consistent with the CBAERo analysis. The change in $L / D$ at trim is due to the change in trim angle produced by the recession, and ranges from $\sim 0.007$ at $U_{\infty}$ $=9 \mathrm{~km} / \mathrm{s}$ to $\sim 0.017$ at $M=6$. There is variation among the different analyses on the order of $18 \%-33 \%$ for the change in trim angle, with the higher percentage variation occurring at the higher Mach number (lower recession) conditions.

The current results validate CBAERo for analyzing aerodynamics due to recession, and provide a basis for computing uncertainties on the CBAERO results. The results do not, however, validate that the prediction of recession by the CBAERO study is correct. The present analysis quantifies the change in aerodynamics, particularly the trim angle of attack, for a specific history of recession. The results, therefore, cannot be interpreted to give an upper bound to the change in trim angle due to recession, due to limitations in the development of the recessed geometries used here.

The study results provide a basis for quantifying uncertainties due to recession for the Orion aerdatabase. The results show no contribution to the uncertainties is necessary for $C_{L}, C_{D}$, or $L / D$. An contribution to the pitching moment uncertainty could be computed from this work. The contribution would require an additional safety factor, due to fact that this sample analysis does not completely bound the problem of finding the maximum likely change in $C_{m}$ due to recession. An additional argument could be made that the uncertainty is one-sided; the pitching moment should always decrease with recession.

With the quantified change in $C_{m}$ and trim angle due to recession and the potential for quantified uncertainties to be applied to the Orion aerodynamic database, this study has provided the CAP team with insight to understand the relative importance of the change in vehicle shape produced by recession. The validation of the CBAERO methodology for predicting the change in pitching moment gives the CAP team a reliable tool for continuing analysis of recession as the program refines the trajectories and moves closer to lunar missions and begins considering Mars missions.

\section{Acknowledgments}

Dave Kinney, Ames Research Center, provided the data for the starting point of this study, with the recessed geometries and aerodynamic data comparison from the CBAERO analysis. He also provided valuable discussions about the interpretation of the current study results. Veronica Hawke, Eloret Corporation, created the high-fidelity CAD geometries based on the CBAERO analysis for the four recessed geometries. Victor Lessard, Genex Systems, developed the meshes for the LAURA computations on the baseline and recessed geometries. 


\section{References}

${ }^{1}$ http://www.nasa.gov/mission_pages/constellation/orion.
${ }^{2}$ CAP Aerodynamics Team, "Orion Aerodynamic Databook, Version 0.25," NASA JSC-63402, CXP-72167, April 2007.
${ }^{3}$ CAP Aerodynamics Team, "Formulation of the Orion Aerodynamic Database," NASA EG-CEV-06-37, rev. 0.25.3, April 2007. 2006.

${ }^{4}$ Kinney, D. J., "CEV Trim Angle of Attack Sensitivity Due to TPS Recession," CAP TN EG-CAP-06-139, draft release,

${ }^{5}$ Kinney, D. J., "CEV Trim Angle of Attack Sensitivity Due to TPS Recession along the Lockheed Martin L/D = 0.3 Skip and Ballistic Trajectories," CAP TN EG-CAP-06-170, 1.0.1 release, 2006.

${ }^{6}$ Wright, M. and TPS ADP Team, "CEV Thermal Protection System (TPS) Margin Management Plan, Draft v6c," Tech. Rep. C-TPSA-A-DOC-7005, 2006.

${ }^{7}$ Wright, M. J., Candler, G. V., and Bose, D., "Data-Parallel Line Relaxation Method for the Navier-Stokes Equations," AIAA Journal, Vol. 36, No. 9, 1998, pp. 1603-1609.

${ }^{8}$ Gnoffo, P. A., Gupta, R. N., and Shinn, J., "Conservation Equations and Physical Models for Hypersonic Air Flows in Thermal and Chemical Nonequilibrium," NASA TP 2867, February 1989.

${ }^{9}$ Gnoffo, P. A., "An Upwind-Biased, Point-Implicit Relaxation Algorithm for Viscous, Compressible Perfect-Gas Flows," NASA TP 2953, February 1990.

${ }^{10}$ Peiro, J., Peraire, J., and Morgan, K., "FELISA Reference Manual and User's Guide, Volume I," University of Wales/Swansea Report CR/821/94, 1994.

${ }^{11}$ Bibb, K. L., Peraire, J., and Riley, C. J., "Hypersonic Flow Computations on Unstructured Meshes," AIAA Paper 97-0625, January 1997.

${ }^{12}$ McDaniel, R., Brown, J., Tang, C., Olejniczak, J., Bahram, P., Dateo, C., Liu, Y., Yoon, S., and Palmer, G., "Best Practices for DPLR CEV Aerothermal Database Solutions, v2," CAP TN Draft, version 2.0, 2007.

${ }^{13}$ Steinbrenner, J. P., Chawner, J. R., and Fouts, C. L., "The GRIDGEN 3D Multiple Block Grid Generation System," Wright Research and Development Center Report TR-90-3022, July 1990.

${ }^{14}$ Wright, M. J. and Candler, G. V., "The Solution of the Navier-Stokes Equations Using Gauss-Seidel Line Relaxation," Computers and Fluids, Vol. 17, No. 1, pp. 135-150.

${ }^{15}$ Menter, F. R., "Two-Equation Eddy-Viscosity Turbulence Models for Engineering Applications," AIAA Journal, Vol. 32, No. 8, 1994, pp. 2558-2564.

${ }^{16}$ Alter, S. J., "The Volume Grid Manipulator (VGM): A Grid Reusability Tool," Tech. Rep. 4772, April 1997.

${ }^{17}$ Gnoffo, P. A., Weilmeunster, K. J., and Greendyke, R. B., "Multiblock Analysis for Shuttle Orbiter Re-Entry Heating from Mach 24 to Mach 12," AIAA Paper 93-2813, July 1993.

${ }^{18}$ Roe, P. L., "Approximate Riemann Solvers, Parameter Vectors, and Difference Schemes," Journal of Computational Physics, Vol. 43, October 1981, pp. 357-372.

${ }^{19}$ Harten, A., "High Resolution Schemes for Hyperbolic Conservation Laws," Journal of Computational Physics, Vol. 49, No. 2, February 1983, pp. 357-393.

${ }^{20}$ Yee, H. C., "On Symmetric and Upwind TVD Schemes," NASA TM 88325, 1986.

${ }^{21}$ Zoby, E. V., Pulsonetti, M., Alter, S., Thompson, R., Meyers, B., Greene, F., Lessard, V., Weilmeunster, J., Hollis, B., and Jentink, T., "Guidelines for Application of the LAURA code for CEV," CAP TN EG-CEV-07-53, 2007.

${ }^{22}$ Park, C., "Review of Chemical-Kinetic Problems of Future NASA Missions, I: Earth Entries," Journal of Thermphysics and Heat Transfer, Vol. 7, No. 3, July 1993, pp. 385-398.

${ }^{23}$ Wright, M. J., Deepak, B., Palmer, G. E., and Levin, E., "Recommended Collision Integrals for Transport Property Computations, Part 1: Air Species," AIAA Journal, Vol. 43, No. 12, pp. 2558-2564.

${ }^{24}$ Jones, W. T., "An Open Framework for Unstructured Grid Generation," AIAA Paper 2002-3192, 2002.

${ }^{25}$ Jones, W. T., "GridEx-An Integrated Grid Generation Package for CFD," AIAA Paper 2003-4129, 2003.

${ }^{26}$ Hänel, D., Schwane, R., and Seider, G., "On the accuracy of Upwind Schemes for the Solution of the Navier-Stokes Equations," AIAA Paper 87-1005, 1987.

${ }^{27}$ Gordon, S. and McBride, B. J., "Computer Program for Calculation of Complex Chemical Equilibrium Composition and Applications: I. Analysis," NASA RP 1311, October 1994.

${ }^{28}$ Bibb, K. L., Gnoffo, P. A., Park, M. A., and Jones, W. T., "Parallel, Gradient Based, Anisotropic Mesh Adaptation for Re-entry Vehicle Configurations," AIAA Paper 2006-3579, June 2006

${ }^{29}$ Bibb, K. L., "CEV CFD Simulation Guidelines for the FELISA Solver," CAP TN EG-CEV-06-30, 0.9.2 release, 2006. 